\title{
Valor del tacto en el diagnóstico de fiebre y de la palidez en el diagnóstico de anemia. Estudio observacional en adultos
}

\author{
C. VALEIRAS, A. CALVO, A. ROCA, F. GUDE*, A. GONZÁLEZ QUINTELA** \\ Unidad Docente de Medicina Familiar y Comunitaria. A Coruña. *Unidad de \\ Epidemiología Clínica y **Servicio de Medicina Interna. Hospital Clínico Universitario \\ de Santiago
}

\author{
DIAGNOSIS OF FEVER BY TOUCH AND DIAGNOSIS OF ANEMIA \\ BY PALLOR. AN OBSERVATIONAL STUDY IN ADULTS
}

\section{RESUMEN}

La estimación por el tacto de la temperatura y la inspección de la presencia de palidez son prácticas habituales durante la exploración de un paciente. Sobre el valor diagnóstico de estos procedimientos existen varios estudios realizados en niños, pero no en adultos.

Objetivo: Conocer el valor diagnóstico del tacto y de la palidez en la estimación de la presencia o no de fiebre y anemia, respectivamente.

Pacientes y métodos: Se incluyeron 600 pacientes (edad media 66 años, intervalo 14-94 años, 58\% varones) ingresados en un Hospital $(n=300)$ o atendidos en su Servicio de Urgencias $(n=300)$. Dos médicos estimaron independiente-mente la presencia de elevación térmica mediante el tacto, así como de palidez cutánea, conjuntival y ungueal. La temperatura axilar medida y con la cifra de hemoglobina circulante se utilizaron como índices de referencia.

Resultados: La especificidad del tacto fue mayor del $90 \%$, tanto para el diagnóstico de una temperatura $\geq 37,2^{\circ} \mathrm{C}$ como $\geq 38^{\circ} \mathrm{C}$, con una sensibilidad sensiblemente menor. La concordancia interobservador fue aceptable (índice Kappa 0,47). Para la prevalencia de fiebre observada (5\%), el valor predictivo negativo fue alto $(99 \%)$, con un valor predictivo positivo mucho menor. De modo similar, la especificidad de la palidez fue elevada, sobre todo para valores de hemoglobina $<10 \mathrm{~g} / \mathrm{dL}$, con una menor sensibilidad. Los mayores valores diagnósticos se observaron con la palidez conjuntival. El índice de concordancia para los diferentes modos de palidez estuvo entre 0,39 y 0,44 . Para la prevalencia encontrada de anemia (hemoglobina $<10 \mathrm{~g} / \mathrm{dL})(9 \%)$, el valor predictivo negativo fue alto $(98 \%)$, con un valor predictivo positivo mucho menor.

Conclusiones: En pacientes adultos atendidos en un hospital, la sensación febril por el tacto y la palidez observada por el médico tienen un valor diagnóstico relativamente escaso. Sin embargo, la estimación de que el paciente tiene una temperatura no elevada o ausencia de palidez hacen improbable la presencia de fiebre y de anemia significativa, respectivamente.

\section{ABSTRACT}

Estimation of fever by touch and of pallor as a sign of anemia are common practices during physical examination. The diagnostic value of these procedures has been extensively studied in children, but there are fewer studies in adults.

Objetive: To evaluate the diagnostic value of physician's estimation of fever and anemia by touch and pallor, respectivelly.

Patients and methods: The study included 600 patients (mean age 66 years, range 14-94 years, 58\% males) admitted to a hospital $(n=300)$ or seen at its Emergency Department $(n=300)$. Two physicians indepen dently estimated the presence of fever by touch, as well as the presence of anemia by pallor (in the skin, nail or conjunctiva). Axillary temperature and peripheral blood hemoglobin were employed as standards for com parison.

Results: Specificity of touch was higher than $90 \%$, both for the diag nosis of axillary temperatures $\geq 37.2^{\circ} \mathrm{C}$ and $\geq 38{ }^{\circ} \mathrm{C}$, with a lower sensiti vity. Interobserver concordance was fair (Kappa index, 0.47). Negative predictive value of touch was also high (99\%) for the observed prevalen ce of fever (5\%), with a very much lower positive predictive value. Simi larly, specificity of pallor was high, particularly for hemoglobin values lower than $10 \mathrm{~g} / \mathrm{dL}$, with a substantially lower sensitivity. The best diag nostic values were obtained with conjunctival pallor. The Kappa index of concordance for the various types of pallor (skin, nail or conjunctiva) ranged between 0,39 and 0,44. Negative predictive value of pallor was high (98\%) for the observed prevalence (9\%) of significant anemia (hemoglobin $<10 \mathrm{~g} / \mathrm{dL}$ ), with a lower positive predictive value.

Conclusions: In adult patients of a hospital, physi-cian's estimation of fever by touch and anemia by pallor have a relatively low diagnostic value. Nevertheless, the estimation that the patient is afebrile or not pale make very improbable the presence of fever and significant anemia, res pectively.

KEY WORDS: Fever. Anemia. Physical examination.

PALABRAS CLAVE: Fiebre. Anemia. Exploración física.

Valeiras C, Calvo A, Roca A, Gude F, González Quintela A. Valor del tacto en el diagnóstico de fiebre y de la palidez en el diagnóstico de anemia. Estudio observacional en adultos. An Med Interna (Madrid) 2002; 19: 120-125.

Trabajo aceptado: 4 de diciembre de 2001

Correspondencia: A. González Quintela. Servicio de Medicina Interna. Hospital Clinico Universitario. C/ Choupana s/n. 15706 Santiago de Compostela. A Coruña. 


\section{INTRODUCCIÓN}

La fiebre es, tras el dolor, la segunda causa de consulta médica, y es percibida como un signo cierto de enfermedad tanto por el paciente como por el médico. Si bien, como es obvio, para su medida es preciso recurrir a métodos objetivos (1), es una práctica habitual estimar de entrada la temperatura corporal por el tacto, tanto por el médico como por el propio paciente o sus acompañantes. Existen estudios previos sobre el valor diagnóstico de la estimación de la temperatura corporal por el tacto (2-10). En algunos de ellos, lo que se evaluó fue la capacidad de reconocer fiebre por el propio paciente (5) o por los padres, en caso de niños $(3,4,6,8,9)$. De hecho, la gran mayoría de estos estudios han sido realizados en pacientes de edad infantil $(2-4,6-9)$ y/o en países tropicales subdesarrollados (4,5,7-9).

La anemia es otro problema clínico habitual. Para su estimación también es preciso recurrir a métodos objetivos, pero la valoración de la palidez cutáneo-mucosa es una parte inicial común de la inspección de un paciente durante una exploración física rutinaria. Existen estudios previos sobre el valor diagnóstico de la palidez en el diagnóstico de anemia (10-18). Sin embargo, al igual que en caso de la fiebre, la gran mayoría de estos estudios han sido realizados en niños y en países subdesarrollados con una alta prevalencia de anemia por malnutrición o enfermedades parasitarias (12-18).

Es una práctica conveniente cuantificar el valor de todos los actos médicos, incluso los más habituales y tradicionalmente aceptados (19). Por ello, el presente estudio fue dirigido a estimar la validez del tacto en el diagnóstico de fiebre, así como de la palidez detectada por el médico en el diagnóstico de anemia en una población adulta de nuestro entorno, en el medio hospitalario.

\section{PACIENTES Y MÉTODOS}

Tipo de estudio: estudio observacional, de pruebas diagnósticas.

Ambito del estudio: pacientes ingresados o que acuden a Urgencias en un Hospital de referencia en el Noroeste Español.

Sujetos a estudio: se incluyeron 600 pacientes. De ellos, 300 se encontraban ingresados en el Servicio de Medicina Interna del Hospital Juan Canalejo de la Coruña. Los restantes 300 fueron seleccionados entre los que acudieron a su Servicio de Urgencias. Se escogieron estas dos situaciones (planta y urgencias) buscando un espectro amplio de pacientes a los cuales podrían ser aplicables los posibles resultados en la práctica clínica. El estudio fue realizado durante tres meses, de Marzo a Mayo del 2000, en días seleccionados al azar necesarios hasta obtener el tamaño de muestra previsto. Fueron incluidos los pacientes ingresados en las unidades del Servicio de Medicina Interna y los que acudieron a Urgencias en los momentos seleccionados. Fueron excluidos los pacientes con deterioro cognitivo incapaces de dar su consentimiento informado para participar en el estudio.

Todos los sujetos incluidos en el estudio eran de raza blanca. Un total de 349 (58\%) eran varones y 251 (42\%) mujeres. La edad media de los sujetos a estudio era 66 años, con una desviación estándar de 18 años, y un intervalo de 14 a 94 años.
Consideraciones éticas: todos los pacientes dieron su consentimiento informado para participar en el estudio.

Instrumentación y determinaciones: todos los pacientes fueron valorados por dos facultativos, ambos residentes de tercer año de la Especialidad de Medicina Familiar y Comunitaria. Los dos valoraron independientemente la presencia de elevación de la temperatura corporal en el sujeto como variable dicotómica (temperatura elevada si/no) mediante palpación sistemática de la frente, cuello, cara anterior del tórax y del abdomen con el dorso de la falange media del $2^{\circ}, 3^{\circ}, 4^{\circ}$ y $5^{\circ}$ dedos. Ambos también estimaron independientemente la palidez en cada sujeto a estudio como variable dicotómica (presencia de palidez si/no) mediante inspección sucesiva de piel, conjuntivas y lecho ungueal, que fueron valoradas separadamente. Los dos investigadores desconocían todos los datos sobre el motivo de ingreso o asistencia del paciente.

Inmediatamente a continuación, un tercer investigador tomó la temperatura axilar al paciente mediante un termómetro de mercurio habitual que se mantuvo en posición durante dos minutos. El mismo investigador fue el encargado de recoger los datos demográficos y clínicos de los pacientes, incluyendo los resultados de un hemograma rutinario, que había sido realizado en un hemoglobinómetro tipo Coulter el mismo dia en los pacientes atendidos en Urgencias, y dentro de los seis dias precedentes en los pacientes ingresados en planta, sin que en éstos últimos hubiese habido transfusión ni signos de sangrado reciente.

Los observadores tomaron su propia temperatura antes de iniciar la toma de registros para descartar alteraciones de su propia temperatura corporal que pudiesen influir en sus estimaciones. Durante el período de estudio la temperatura del centro estuvo entorno a los $21^{\circ} \mathrm{C}$. Los datos se tomaron en todos los casos entre las 16 y las 18 horas, valorando a los pacientes con la luz natural, en el caso de pacientes ingresados, y con luz artificial en el Servicio de Urgencias.

Análisis estadístico: para el diagnóstico de elevación térmica, se tomó como patrón de referencia la temperatura axilar, en dos niveles: febrícula (temperatura $\geq 37,2^{\circ} \mathrm{C}$ ), y fiebre (temperatura $\geq 38^{\circ} \mathrm{C}$ ). Se calculó la sensibilidad, especificidad, valor predictivo positivo, valor predictivo negativo y precisión diagnóstica global del tacto del médico (20), al igual que la razón de verosimilitud positiva (sensibilidad/[1-especificidad]) y negativa ([1-sensibilidad/especificidad]) (21). De modo similar, en el diagnóstico de anemia se tomó como patrón de referencia la cifra de hemoglobina del hemograma, en dos niveles: hemoglobina menor de $12 \mathrm{~g} / \mathrm{dL}$ (anemia) y hemoglobina menor de $10 \mathrm{~g} / \mathrm{dL}$ (anemia significativa). Las proporciones obtenidas se expresan como porcentajes, aproximados a sus valores enteros. Tanto en el diagnóstico de fiebre como en el de anemia, se presentan los valores obtenidos por los dos investigadores. La concordancia interobservador se evaluó mediante el coeficiente Kappa (22). Dado que los valores predictivos varían en función de la prevalencia del fenómeno, se estimaron estos valores predictivo y negativo en función de la sensibilidad y especificidad observadas para diferentes probabilidades previas (prevalencias) de acuerdo con el teorema de Bayes (23). Los pacientes ingresados en planta y atendidos en Urgencias fueron analizados conjuntamente. En el valor de la palidez para el diagnóstico de anemia, se analizaron además separadamente los pacientes vistos en planta y los pacientes vistos en Urgencias, por la diferencia en las condiciones de observación (calidad de luz). 


\section{RESULTADOS}

\section{VALOR DEL TACTO EN EL DIAGNÓSTICO DE FIEBRE}

La temperatura axilar media de los pacientes estudiados fue $36,4^{\circ} \mathrm{C}$, con una desviación estándar de $0,8^{\circ} \mathrm{C}$ y un intervalo de $35-39^{\circ} \mathrm{C}$. Un total de 74 pacientes $(12 \%)$ presentaba elevación térmica (al menos, febrícula) y, de ellos, 29 pacientes $(5 \%)$ presentaban fiebre.

Los datos de sensibilidad, especificidad, valor predictivo positivo, valor predictivo negativo, precisión global, razón de verosimilitud positiva y razón de verosimilitud negativa de la estimación subjetiva del médico en el diagnóstico de elevación térmica en el paciente se recogen en la tabla I. Destaca la elevada especificidad y valor predictivo negativo de la estimación del médico, tanto en los casos de febrícula como de fiebre. La especificidad estuvo por encima del $90 \%$ en ambos casos. El valor predictivo negativo fue particularmente alto (cercano al 100\%) en los casos de fiebre, de modo que la sensación por parte del médico de que el paciente no presentaba temperatura elevada prácticamente descartó que el sujeto tuviera $38^{\circ} \mathrm{C}$ o más de temperatura axilar (Tabla I). La sensibilidad del test fue mucho menor, al igual que el valor predictivo positivo, de modo que menos de un tercio de los casos en los que el médico percibió que el paciente tenía temperatura elevada, existía fiebre en la realidad (Tabla I). De acuerdo con la razón de verosimilitud positiva, en los pacientes con febrícula o fiebre es unas 8-9

\section{TABLA I}

VALOR DIAGNÓSTICO DEL TACTO DEL MÉDICO (TEM PERATURA ELEVADA SÍ/NO ) EN RELACIÓN A LA TEM PERATURA M EDIDA EN EL PACIENTE

\begin{tabular}{|c|c|c|}
\hline & $\begin{array}{r}\text { Febrícula } \\
\left(\mathrm{T}^{\mathrm{a}} \geq 37,2 \stackrel{\circ}{ } \mathrm{C}\right) \\
\end{array}$ & $\begin{array}{r}\text { Fiebre } \\
\left(\mathrm{T}^{\mathrm{a}} \geq 38 \stackrel{\circ}{ } \mathrm{C}\right)\end{array}$ \\
\hline $\begin{array}{r}\text { Sensibilidad (1) } \\
(2)\end{array}$ & $\begin{array}{l}51 \%(38 / 74) \\
65 \%(48 / 74)\end{array}$ & $\begin{array}{ll}76 \% & (22 / 29) \\
93 \% & (27 / 29)\end{array}$ \\
\hline $\begin{array}{r}\text { Especificidad (1) } \\
(2)\end{array}$ & $\begin{array}{l}94 \%(495 / 526) \\
93 \%(487 / 526)\end{array}$ & $\begin{array}{ll}92 \% & (524 / 571) \\
90 \% & (511 / 571)\end{array}$ \\
\hline $\begin{array}{r}\text { V.P. positivo (1) } \\
(2)\end{array}$ & $\begin{array}{l}55 \%(38 / 69) \\
55 \%(48 / 87)\end{array}$ & $\begin{array}{ll}32 \% & (22 / 69) \\
31 \% & (27 / 87)\end{array}$ \\
\hline $\begin{array}{r}\text { V.P. negativo (1) } \\
\text { (2) }\end{array}$ & $\begin{array}{l}93 \%(495 / 531) \\
95 \%(487 / 513)\end{array}$ & $\begin{array}{ll}99 \% & (524 / 531) \\
99 \% & (511 / 513)\end{array}$ \\
\hline $\begin{array}{l}\text { Precisión } \\
\text { global }\end{array}$ & $\begin{array}{ll}89 \% & (533 / 600) \\
89 \% & (535 / 600)\end{array}$ & $\begin{array}{ll}91 \% & (546 / 600) \\
90 \% & (538 / 600)\end{array}$ \\
\hline $\begin{array}{r}\text { R.V. positiva (1) } \\
\text { (2) }\end{array}$ & $\begin{array}{l}8,7 \\
8,8\end{array}$ & $\begin{array}{l}9,3 \\
8,9\end{array}$ \\
\hline $\begin{array}{r}\text { R.V. negativa (1) } \\
\text { (2) }\end{array}$ & $\begin{array}{l}0,5 \\
0,4\end{array}$ & $\begin{array}{l}0,3 \\
0,1\end{array}$ \\
\hline
\end{tabular}

(1): observador $n$ ㅇ 1; (2): observador $n$ ㅇ 2; V.P.: valor predictivo; R.V.: razón de verosimilitud. veces más probable que el médico perciba elevación térmica por el tacto que en los pacientes con temperatura normal. Los resultados fueron similares en los dos inves-tigadores independientes (Tabla I). El índice Kappa de concordancia global entre los dos investigadores fue 0,47.

\section{VALOR DE LA PALIDEZ EN EL DIAGNÓSTICO DE ANEMIA}

La cifra media de hemoglobina en los pacientes estudiados fue 13,2 g/dL, con una desviación estándar de $2,2 \mathrm{~g} / \mathrm{dL}$ y un intervalo de 5,5-20 g/dL. Un total de 139 pacientes (23\%) presentaba una cifra de hemoglobina menor de $12 \mathrm{~g} / \mathrm{dL}$ y, de ellos, 51 pacientes (9\% del total) presentaban una hemoglobina menor de $10 \mathrm{~g} / \mathrm{dL}$.

Los resultados del valor diagnóstico de la palidez se reflejan en la tabla II. Para las tres categorías de palidez (cutánea, conjuntival y ungueal), la especificidad fue mayor que la sensibilidad, y el valor predictivo negativo mayor que el positivo. La ausencia de palidez en las zonas estudiadas descartó acertadamente la presencia de anemia significativa $(\mathrm{Hb}<10 \mathrm{~g} / \mathrm{dL})$ en la mayoría de los casos. La mejor precisión diagnóstica, el mejor valor predictivo negativo, y los mayores valores de razón de verosimilitud positiva se observaron con la palidez conjuntival (Tabla II). La sensibilidad del test fue particularmente escasa. Así, tomando como valor de refe-rencia una hemoglobina de $10 \mathrm{mg} / \mathrm{dL}$, los dos médicos sólo detectaron que el paciente tenía palidez cutánea, ungueal o conjuntival en el 67\% (34/51) y en el 77\% (39/51) de los casos, respectivamente. De modo similar, el hecho de que dos médicos estimasen que el paciente tenía palidez cutánea, ungueal o conjuntival sólo fue concordante con que el paciente tuviese una hemoglobina menor de $12 \mathrm{~g} / \mathrm{dL}$ (valor predictivo positivo) en el $44 \%$ (75/171) y en el $37 \%$ (91/244) de los casos.

Los resultados obtenidos por los dos investigadores independientes fueron similares (Tabla II). Los índices Kappa de concordancia entre los dos investigadores fueron 0,39, 0,41, y 0,44 para la palidez ungueal, cutánea y conjuntival, respectivamente. Cuando se compararon los resultados obtenidos en cuanto al valor de la palidez conjuntival en los pacientes ingresados en planta (examinados con luz natural) y en urgencias (con luz artificial) los resultados fueron similares (Tabla III).

\section{DISCUSIÓN}

De acuerdo con los resultados del estudio, el tacto del médico tiene una elevada especificidad y valor predictivo negativo para el diagnóstico de elevación térmica (febrícula o fiebre) en pacientes adultos en el medio hospitalario. Sin embargo, la sensibilidad y el valor predictivo positivo de la sensación del médico son más bajos. La razón de verosimilitud positiva estuvo entre 8 y 9 , niveles que se consideran indicativos de una moderada utilidad del test (21). La concordancia obtenida entre dos observadores de parecida experiencia y formación puede calificarse como aceptable (22). Estos resultados son algo diferentes a los referidos en estudios previos similares, en los que se encontró que el tacto del médico tenía una menor especificidad y sobre todo una mayor sensibilidad que en el presente estudio, de hasta el $94 \%(8,9)$. Existen varios factores que pueden contribuir a estas diferencias. En primer lugar, el nivel elegido para 
TABLA II

VALOR DIAGNÓSTICO DE LA INSPECCIÓN (PRESENCIA DE PALIDEZ SÍ/NO) EN RELACIÓN A LA CIFRA DE HEM OGLOBINA DEL PACIENTE

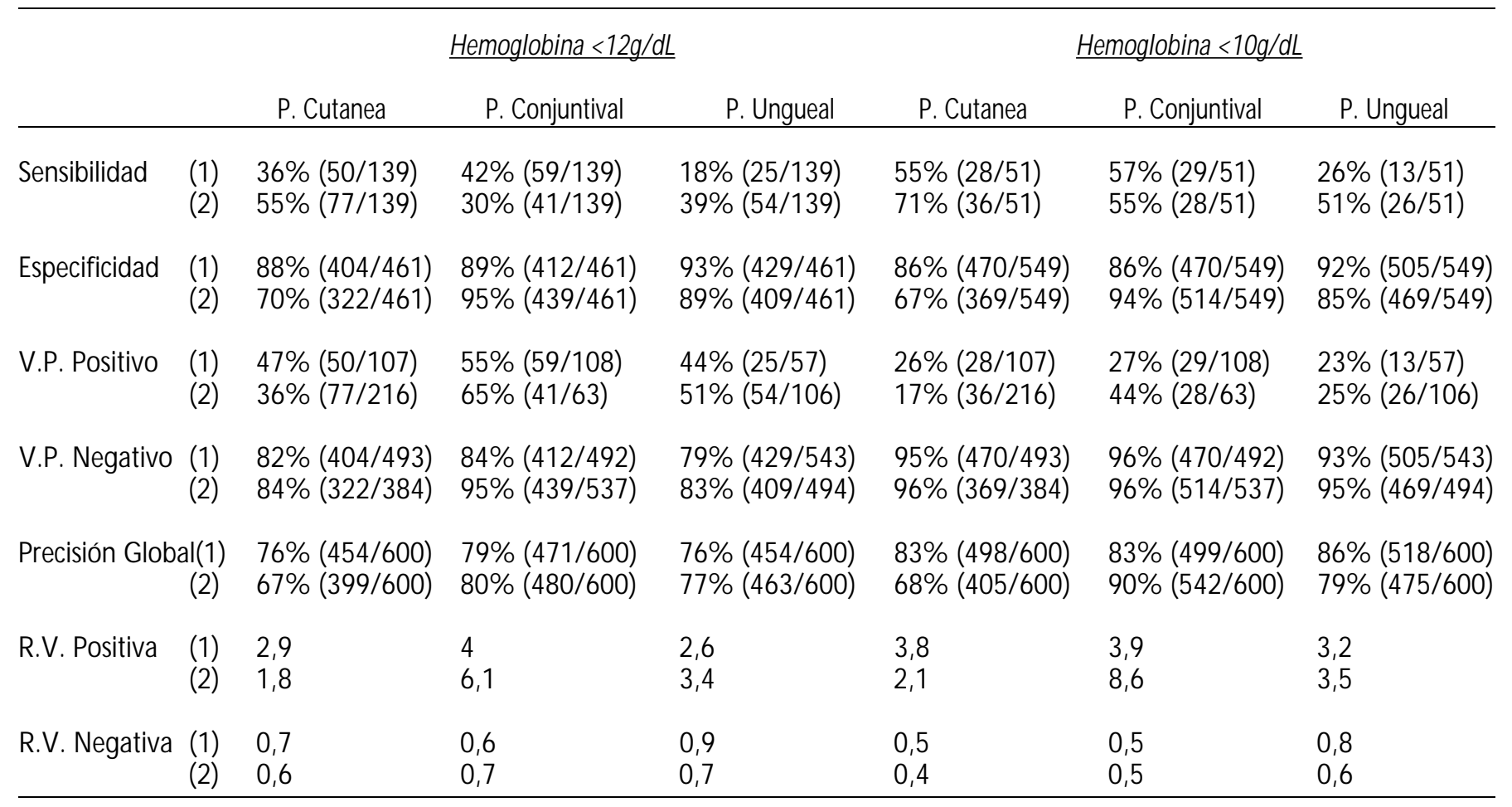

P.: Palidez; (1): observador no 1; (2): observador no 2; V.P.:valor predictivo; R.V.: razón de verosimilitud.

TABLA III

COM PARACIÓN DE VALOR DIAGNÓSTICO DE LA PALIDEZ CONJUNTIVAL EN PACIENTES ATENDIDOS EN PLANTA (LUZ NATURAL) Y URGENCIAS (LUZ ARTIFICIAL)

\begin{tabular}{|c|c|c|c|c|c|}
\hline & & Hemoglo & $12 \mathrm{~g} / \mathrm{dL}$ & Hemoglob & $.0 \mathrm{~g} / \mathrm{dL}$ \\
\hline & & Planta & Urgencias & Planta & Urgencias \\
\hline Sensibilidad & $\begin{array}{l}(1) \\
(2)\end{array}$ & $\begin{array}{ll}45 \% & (43 / 96) \\
33 \% & (32 / 64)\end{array}$ & $\begin{array}{l}37 \%(16 / 43) \\
21 \%(9 / 43)\end{array}$ & $\begin{array}{ll}57 \% & (21 / 37) \\
60 \% & (22 / 37)\end{array}$ & $\begin{array}{l}57 \%(8 / 14) \\
43 \%(6 / 14)\end{array}$ \\
\hline Especificidad & $\begin{array}{l}(1) \\
(2)\end{array}$ & $\begin{array}{l}84 \%(172 / 204) \\
92 \%(188 / 204)\end{array}$ & $\begin{array}{l}93 \%(240 / 257) \\
98 \%(251 / 257)\end{array}$ & $\begin{array}{ll}80 \% & (209 / 263) \\
90 \% & (237 / 263)\end{array}$ & $\begin{array}{l}91 \%(261 / 286) \\
97 \%(277 / 286)\end{array}$ \\
\hline V.P: positivo & $\begin{array}{l}(1) \\
(2)\end{array}$ & $\begin{array}{l}57 \%(43 / 75) \\
67 \%(32 / 48)\end{array}$ & $\begin{array}{l}48 \%(16 / 33) \\
60 \%(9 / 15)\end{array}$ & $\begin{array}{ll}28 \% & (21 / 75) \\
46 \% & (22 / 48)\end{array}$ & $\begin{array}{l}24 \%(8 / 33) \\
40 \%(6 / 15)\end{array}$ \\
\hline V.P. negativo & $\begin{array}{l}(1) \\
(2)\end{array}$ & $\begin{array}{l}76 \%(172 / 225) \\
75 \%(188 / 252)\end{array}$ & $\begin{array}{l}90 \%(240 / 267) \\
88 \%(251 / 285)\end{array}$ & $\begin{array}{l}93 \%(209 / 225) \\
94 \%(237 / 252)\end{array}$ & $\begin{array}{l}98 \%(261 / 267) \\
97 \%(277 / 285)\end{array}$ \\
\hline Precisión global & $\begin{array}{l}(1) \\
(2)\end{array}$ & $\begin{array}{l}72 \%(215 / 300) \\
73 \%(220 / 300)\end{array}$ & $\begin{array}{l}85 \%(256 / 300) \\
87 \%(260 / 300)\end{array}$ & $\begin{array}{l}77 \%(230 / 300) \\
86 \%(259 / 300)\end{array}$ & $\begin{array}{l}90 \%(269 / 300) \\
94 \%(283 / 300)\end{array}$ \\
\hline R.V. positiva & $\begin{array}{l}(1) \\
(2)\end{array}$ & $\begin{array}{l}2,9 \\
4,3\end{array}$ & $\begin{array}{l}5,6 \\
9,1\end{array}$ & $\begin{array}{l}2,8 \\
6,0\end{array}$ & $\begin{array}{l}6,6 \\
13,8\end{array}$ \\
\hline R.V. negativa & $\begin{array}{l}(1) \\
(2)\end{array}$ & $\begin{array}{l}0,7 \\
0,7\end{array}$ & $\begin{array}{l}0,7 \\
0,8\end{array}$ & $\begin{array}{l}0,5 \\
0,4\end{array}$ & $\begin{array}{l}0,5 \\
0,6\end{array}$ \\
\hline
\end{tabular}

(1): observador no 1; (2): observador no 2. V.P.: valor predictivo; R.V.: razón de verosimilitud. 
definir la elevación térmica, variable según los diferentes estudios. En los estudios que demostraron una más alta sensibilidad en el diagnóstico de fiebre por el tacto, el dintel para definir una temperatura corporal elevada se estableció en $37,8^{\circ} \mathrm{C}$ axilar (9). En el presente estudio, se escogieron dos niveles para el análisis, $37,2^{\circ} \mathrm{C}$ (febrícula) y $38^{\circ} \mathrm{C}$ (tradicionalmente considerada como fiebre). Como era de esperar, la sensibilidad del tacto fue mayor cuando el nivel para definir temperatura elevada se estableció en $38^{\circ} \mathrm{C}$ en lugar de $37,2^{\circ} \mathrm{C}$, sin modificarse apreciablemente la especificidad. Los estudios que utilizan la temperatura rectal como valor de referencia (8) son menos comparables por la diferencia bien conocida entre las temperaturas axilar y rectal (1). En segundo lugar, hay que tener en cuenta que la casi totalidad de los estudios similares realizados hasta la fecha lo han sido en niños, y en zonas tropicales con temperatu-ras ambientales más altas $(8,9)$, mientras que el presente estudio fue realizado en adultos con una edad media avanzada, y en diferentes condiciones climáticas. En tercer lugar, la prevalencia de fiebre, que en nuestro estudio fue más baja que en estudios similares ya mencionados realizados en niños $(8,9)$, puede modificar los valores predictivo positivo y negativo del test, aunque no así tanto la sensibilidad y especificidad (20). Partiendo de estos últimos valores, la palpación mantendría un alto valor predictivo negativo (no así el valor predictivo positivo) en un amplio abanico de prevalencias (Tabla IV). Por último, hay que tener en cuenta la variación interobservador, siempre presente, si bien la concordancia entre dos investigadores independientes en nuestro estudio fue aceptable. Incluso en estudios previos la concordancia entre médicos y familiares del paciente (sobre todo madres de niños) también fue satisfactoria $(8,9)$.

Algo similar ocurre con la estimación de palidez por parte del médico, que demuestra una elevada especificidad y valor predictivo negativo para el diagnóstico de anemia en pacientes adultos en el medio hospitalario, junto a una menor sensibilidad $\mathrm{y}$ valor predictivo positivo. El elevado valor predictivo negativo

\section{TABLA IV}

ESTIM ACIÓ N M EDIANTE EL TEO REM A DE BAYES DE LOS VALO RES PREDICTIVOS DEL TACTO EN EL DIAGNÓ STICO DE FIEBRE $\left(T^{A} \geq 38^{\circ} \mathrm{C}\right)$ Y DE LA PALIDEZ CONJUNTIVAL EN EL DIAGNÓSTICO DE ANEM IA SIGNIFICATIVA (HB<10 G/DL) EN FUNCIÓN DE LA SENSIBILIDAD Y ESPECIFICIDAD O BSERVADAS PARA DIFERENTES PRO BABILIDADES PREVIAS (PREVALENCIAS)

\begin{tabular}{lcccc}
\hline Prevalencia & \multicolumn{2}{c}{ Fiebre } & \multicolumn{2}{c}{ Anemia } \\
\hline & $\underline{\text { V.P.P. }}$ & $\underline{\text { V.P.N. }}$ & $\underline{\text { V.P.P. }}$ & $\underline{\text { V.P.N. }}$ \\
$20 \%$ & $69 \%$ & $98 \%$ & $68 \%$ & $89 \%$ \\
$10 \%$ & $49 \%$ & $99,1 \%$ & $49 \%$ & $95 \%$ \\
$5 \%$ & $32 \%$ & $99,6 \%$ & $31 \%$ & $98 \%$ \\
$1 \%$ & $8 \%$ & $99,9 \%$ & $8 \%$ & $99,5 \%$ \\
1 por mil & $0,9 \%$ & $99,99 \%$ & $0,9 \%$ & $99,9 \%$ \\
\hline
\end{tabular}

Como valores de sensibilidad y especificidad de referencia para la estimación de los valores predictivos se han tomado los del observador $\mathrm{n} 02$. V.P.P.: valor predictivo positivo; V.P.N.: valor predictivo negativo se mantendría para prevalencias mayores y menores que la observada (Tabla IV). Los valores de la razón de verosimilitud positiva estuvieron mayoritariamente comprendidos entre 2 y 5 , comparativamente menores que los observados para la fiebre y el tacto. Los resultados obtenidos son similares a los referidos en estudios previos (10-17). Las diferencias observables, sobre todo la mayor sensibilidad referida en algunos estudios $(15,17)$ se pueden explicar por el nivel más bajo de hemoglobina elegido para la definición de anemia. Los niveles elegidos para el análisis en el presente estudio fueron $12 \mathrm{~g} / \mathrm{dL}$ y $10 \mathrm{~g} / \mathrm{dl}$, que definirían anemia en ambos sexos, en el segundo caso significativa. Aunque los niveles para definir anemia sean diferentes en la mujer y en el varón, se asumió que el grado de palidez para un nivel determinado de hemoglobina sería independiente del sexo del paciente. Como era de esperar, la sensibilidad de la palidez aumentó para niveles más bajos de hemoglobina circulante, sin modificarse apreciablemente la especificidad. Existió una cierta variación interobservador, si bien la concordancia entre dos investigadores independientes en nuestro estudio se puede calificar de aceptable (22). La mejor precisión diagnóstica y la mejor concordancia interobservador se observaron con la palidez conjuntival respecto a la cutánea o ungueal. Clásicamente, se asume que la inspección de la coloración cutánea es más válida con luz natural. En nuestro estudio, sin embargo, el valor de la palidez conjuntival fue similar en pacientes atendidos en planta (con luz natural) y en urgencias (con luz artificial), tendiendo incluso a ser mejor la precisión diagnóstica en los pacientes examinados en urgencias con luz artificial. Algo parecido ocurrió con la palidez cutánea y ungueal (datos no presentados). De acuerdo con algunos estudios, el rendimiento de la inspección de palidez se puede incrementar comparando el color de la piel o mucosa con una escala de color validada, lo que puede hacer significativa su eficiencia en áreas subdesarrolladas donde la práctica de un hemograma puede resultar difícil (18). Hay que tener en cuenta, al igual que en el caso de la fiebre, que la casi totalidad de los estudios similares publicados hasta la fecha fueron realizados en niños de zonas tropicales del Tercer Mundo, ya que la edad y la diferente coloración de la piel podrían hacer variar los resultados de la observación. El color normal de la piel depende de varios pigmen-tos, de los cuales la cantidad de melanina, de origen genético e inducible por la luz solar, puede enmascarar la palidez. Hay que destacar además que la media de edad de los pacientes del estudio fue elevada, pues la piel se ve afectada por el envejecimiento, tendiendo a ser más opaca y pálida por menor vascularización de la dermis (24).

La medición objetiva de la temperatura es obligada en toda exploración física rutinaria. Obviamente, el tacto no puede sustituir al termómetro. De modo similar, la observación no puede reemplazar a un hemograma, que es una técnica barata, reproducible y con un riesgo mínimo. No era éste el objetivo del estudio, sino cuantificar el valor de unas prácticas habituales. En resumen, y de acuerdo con nuestros datos, en pacientes adultos del medio hospitalario, cuando el médico detecta por el tacto que la temperatura del paciente no está elevada, o detecta por la inspección que no existe palidez, hace improbable la presencia de fiebre y anemia significativa, respectivamente. Por contra, la sensación de que el paciente tiene fiebre o que presenta palidez tiene un valor relativamente escaso. La estimación de la fiebre por el tacto y de la anemia por la inspección podrían utilizarse como herramientas de screening en determinadas circunstancias, más que como tests diagnósticos. 


\section{Bibliografía}

1. Shann F, Mckenzie A. Comparison of rectal, axillary and forehead temperatures. Arch Pediatr Adolesc Med 1996; 150: 74-8.

2. Bergeson PS, Stienfeld HJ. How dependable is palpation as a screening method for fever? Can touch substitute for thermometer readings? Clin Pediatr 1974; 13: 350-1.

3. Banco L, Veltri D. Ability of mothers to subjectively assess the presence of fever in their children. Am J Dis Child 1984; 138: 976-8.

4. Singhi S, Sood V. Reliability of subjective assessment of fever by mothers. Indian Pediatr 1990; 27: 811-5.

5. Einterz EM, Bates ME. Fever in Africa: do patients know when they are hot? Lancet 1997; 350: 781

6. Grabeto JW, Soglin DF. Maternal screening of childhood fever by palpation. Pediatr Emerg Care 1996; 12: 183-4.

7. Schwartz N, Guendelman S, English P. Thermometer use among Mexican immigrant mot-hers in California. Soc Sci Med 1997; 45: 1315-23.

8. Nwanyanwu OC, Ziba C, Redd SC, Luby SP. Palpation as a method of ferer determination in Malawian children who are less than 5 years old: how reliable is it? Ann Trop Med Parasitol 1997; 91: 359-63.

9. Whybrew K, Murray M, Morley C. Diagnosing fever by touch: observational study. Br Med J 1998; 317: 321.

10. Hung OL, Kwon NS, Cole AE, Dacpano GR, Wu T, Chiang WK, et al. Evaluation of the physician's ability to recognize the presence or absence of anemia, fever, and jaundice. Acad Emerg Med 2000; 7: 146-56.

11. Gjorup T, Bugge PM, Hendriksen C, Jensen AM. A critical evaluation of the clinical diagnosis of anemia. Am J Epidemiol 1986; 124: 657-65.

12. Wurapa FK, Bulsara MK, Boatin BA. Evaluation of conjunctival pallor in the diagnosis of anaemia. J Trop Med Hyg 1986; 89: 33-6.

13. Sdepanian VL, Silvestrini WS, de Morais MB. Diagnostic limitations of the physical examination in the identification of children with anemia. Rev Assoc Med Bras 1996; 42: 169-74.

14. Ekunwe EO. Predictive value of conjunctival pallor in the diagnosis of anemia. West Afr J Med 1997; 16: 246-50.

15. Kalter HD, Burnham G, Kolstad PR, Hossain M, Schillinger JA, Kahn NZ, et al. Evaluation of clinical signs to diagnose anaemia in Uganda and Bangladesh, in areas with and without malaria. Bull World Health Organ 1997; 75 (Suppl 1): 103-11.

16. Weber MW, Kellingray SD, Palmer A, Jaffar S, Mulholland EK, Greenwood BM. Bull World Health Organ 1997; 75 (Suppl 1): 113-8.

17. Stoltzfus RJ, Edward-Raj A, Dreyfuss ML, Albonico M, Montresor A, Dhoj Thapa M, et al. Clinical pallor is useful to detect severe anemia in populations where anemia is prevalent and severe. $J$ Nutr 1999; 129:1675-1681.

18. Montresor A, Albonico M, Khalfan N, Stoltzfus RJ, Tielsch JM, Chwaya HM, et al. Field trial of a hemoglobin colour scale: an effective tool to detect anaemia in pres-chool children. Trop Med Int Health 2000; 5:1 29-33.

19. Sackett DL, Richardson WS, Rosenberg W, Haynes R. Medicina Basada en la Evidencia. Ed. Churchill Livingstone: Madrid, 1997.

20. Goldman L. Quantitative aspects of clinical reasoning. En: Fauci AS, Braunwald E, Isselbacher KJ, Wilson JD, Martin JB, Kasper DL, Hauser SL, Longo DL, eds. Harrison's Principles of Internal Medicine. McGraw-Hill: Nueva York, 1998; 9-14.

21. Jaeshcke R, Guyatt GH, Sackett DL. How to use an article about a diagnostic test. What are the results and will they help me in caring for my patients? JAMA 1994; 271: 703-7.

22. Fleiss JL. Statistical methods for rates and proportions. John Wiley and Sons: Nueva York, 1981.

23. Domenech Massons JM. Fundamentos de diseño y estadística. UD 3: Fundamentos de la teoría de la probabilidad. Pruebas diagnósticas. Ed. Signo: Barcelona, 2001.

24. Fenske NA, Lober CW. Structural and functional changes of normal aging skin. J Am Acad Dermatol 1986; 15: 571-85. 